\section{Inferring the quality of hospital treatment for COPD by mortality; caution is needed}

\author{
M D L Morgan
}

COPD is one of the top five killer conditions in the developed and the developing world. In the UK it is currently ranked number four but set to rise to number three in the rankings of years of life lost behind stroke and ischaemic heart disease. $^{1}$ The natural history of COPD is characterised by progressive decline in airway function accompanied by the unpredictable occurrences of exacerbations. The importance of exacerbations in the natural history of the condition is now understood and embedded in recent clinical guidelines. ${ }^{2}$ Some exacerbations will result in a hospital admission and we know that the need for hospital admission signals a poor prognosis. Half of all patients admitted to hospital with an exacerbation of COPD will die within 4 years. ${ }^{3}$ Those patients who are admitted to hospital are also associated with increased risk with previous audit studies showing an in-hospital mortality rate of $7.4 \% .^{4}$ It is logical to think that improvements in hospital care of patients admitted with acute exacerbation would result in a reduction of mortality and that this would be some reflection on the quality of the service provided to the patient. In other circumstances, the use of the hospital mortality rate as a reflection of service quality would seem an excellent proxy. No one would doubt that improved survival after acute myocardial infarction or lower than average mortality rates for elective surgery would be a measure of the quality of service provided by the hospital. Walker et al have examined whether the mortality within 30 days of admission to hospital for an acute exacerbation of COPD can be used to define the quality of COPD care provided by hospitals. ${ }^{5}$ The study examined Hospital Episode Statistics data from 150 acute hospitals in England over two consecutive years in 2006/2007 and 2007/ 2008. This generated almost 125000 admissions each year and the 30 day mortality was examined for in-year and

Correspondence to Professor M D L Morgan, Department of Respiratory Medicine, University Hospitals of Leicester, Glenfield Hospital, Leicester LE3 9QP, UK; mike.morgan@uhl-tr.nhs.uk between-year variability. The datasets were robust and investigators were able to correct for age, gender, comorbidity, deprivation and the involvement of a specialist respiratory physician. They were not however, able to correct for severity as forced expiratory volume in one second since the data was not available. The results of this examination shed new light on important issues. First, the 30 day mortality rate is high with an overall figure of $11.3 \%$. The mean year-by-year mortality rate was similar but the wide variation between years so extreme that it cannot be useful as a means to make comparison between hospitals or read much into individual centre variation. In addition it appears as if the provision of specialist consultant care makes no difference to crude mortality and curiously, greater deprivation score is associated with better prognosis.

At first sight, these results would seem disappointing and illogical. How can variability in the quality of acute hospital care fail to have an impact on mortality? As the authors acknowledge, acute exacerbation of COPD is part of a chronic and deceptively complex condition which is difficult to gauge. It is just not the same as dealing with single events such as pneumonia, myocardial infarction or a surgical procedure. COPD is a chronic condition that is characterised by unpredictable exacerbations and the end of life cannot be predicted in a similar way to malignant diseases' making it difficult to plan care that takes account of the expectation of death. Most patients will survive a hospital admission for COPD and expect to recover approximately to the preadmission functional status until the next exacerbation. The elegant paper from Suissa has shown us that after one hospital admission there might be a honeymoon period followed by repeated and increasingly frequent admissions until death finally ensues. ${ }^{3}$ In most cases the hospital will be dealing with a patient at the end of an inevitable chain of events with no hope of reversing the condition. Furthermore as we improve acute hospital care, with more patients receiving NIV to correct respiratory failure, we may simply be deferring death until the next admission or perhaps the one after. That is not to say that we should not examine hospital mortality since there are some patients that you would not expect to die and there is an argument for being selective. These might include patients with only moderate airway dysfunction (GOLD I, II), those without severe disability (MRC 2, 3) and those who have clear correctable factors such as pneumonia. Also patients who are on their first admission and those who are previously undiagnosed would be expected to survive. Examination of mortality figures in these patients may potentially be a more accurate reflection of the quality of care.

Crude mortality rates from a particular geographical area may tell us more about the whole health community approach to COPD. Unscheduled admissions to hospital may be influenced by factors other than the severity of the disease itself. It is of interest that Walker et al identified the fact that living in a deprived area was associated with higher hospital admission rates and a lower mortality. This implies that lack of access to good quality primary care services or a structured community care programme will increase hospital usage. In that case, areas with good community care might well perversely show a high mortality rate because the fitter patients are dealt with by services outside hospital.

These arguments suggest that hospital mortality may not be an accurate reflection of the quality of hospital care. In their study, Walker et al have tried to make sense of the best data they could get but they are still vulnerable to the inaccuracy of clinical coding. Their study also lacks the power of a prospective data collection exercise. Nevertheless they have strongly suggested that crude mortality figures are not a good reflection of the care received for the acute episode of COPD. If that is the case, then what metrics are available to judge the quality of care? Although they do not really develop this theme the authors do suggest that length of stay or bed days per head of population corrected for local COPD prevalence may say something about the efficiency of the whole community approach to COPD. There are some other options. Hospital care for patients with COPD may sometimes be necessary even in the most utopian of healthcare systems but to judge performance on mortality alone would be to miss the point. In the best system of community care only the most severe cases might need hospital attention. Others have suggested ways to assess the quality of hospital care. These 
might include process measures such as timely assessment, the availability of noninvasive ventilation and the involvement of a respiratory specialist. ${ }^{6}$ One indicator of good quality care is the completion of a COPD care or discharge bundle. ${ }^{7}$ These checklists record the fact that the patients have received the best evidence-based care and are signposted to pulmonary rehabilitation and smoking cessation services. Patient-reported experience measures are also likely to gain popularity as a component of quality. The acute hospital care of patients with advanced COPD is under scrutiny largely because it is the biggest component of healthcare spend in this condition. While no one would argue that hospital care should never be required or that admissions should be avoided at all costs, hospital care is not going to be the area that has a significant impact on overall COPD mortality. Once the point is reached where emergency admissions to hospital occurs, then mortality as a general outcome becomes less amenable. If we want to prevent premature deaths from COPD then the efforts must start with early detection of the disease before symptoms have developed or hospital admissions start to occur. Hospital mortality following admission from an acute exacerbation of COPD is too simplistic a measure and probably cannot be standardised enough to make it useful. It also, perhaps, detracts from the proper focus of attention on prevention and early detection. It is not quite shutting the stable door after the horse has bolted, but not far removed.

\section{Competing interests None.}

Provenance and peer review Commissioned; internally peer reviewed.

To cite Morgan MDL. Thorax 2013;68:897-898.

Published Online First 28 June 2013

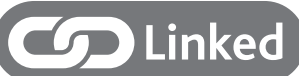

- http://dx.doi.org/10.1136/thoraxjnl-2012-202365

Thorax 2013;68:897-898.

doi:10.1136/thoraxjnl-2013-203717

\section{REFERENCES}

1 Murray CJL, Richards MA, Newton JN, et al. UK health performance: findings of the Global Burden of Disease Study 2010. Lancet 2013;381:997-1020.

2 Vestbo J, Hurd SS, Agusti AG, et al. Global strategy for the diagnosis, management, and prevention of chronic obstructive pulmonary disease: GOLD executive summary. Am J Respir Crit Care Med 2013;187:347-65.

3 Suissa S, Dell'Aniello S, Ernst P. Long-term natural history of chronic obstructive pulmonary disease: severe exacerbations and mortality. Thorax 2012;67: 957-63.

4 Price LC, Lowe D, Hosker HS, et al. UK National COPD Audit 2003: impact of hospital resources and organisation of care on patient outcome following admission for acute COPD exacerbation. Thorax 2006:61:837-42.

5 Walker $\mathrm{P}$, Thompson E, Crone $\mathrm{H}$, et al. Use of mortality within 30 days of a COPD hospitalisation as a measure of COPD care in UK hospitals. Thorax 2013;68:968-70.

6 NHS Improvements Lung Team. Managing exacerbations in chronicobstructive pulmonary disease (COPD): A secondary care toolkitThe ingredients for success. NHS Improvements 2013. http://www. improvement.nhs.uk/documents/COPD_Secondary_ Toolkit.pdf

7 Hopkinson NS, Englebretsen C, Cooley N, et al. Designing and implementing a COPD discharge care bundle. Thorax 2012;67:90-2. 